Document downloaded from:

http://hdl.handle.net/10251/98268

This paper must be cited as:

Manuel Pineda-Sanchez; Sapena-Bano, A.; Pérez-Cruz, J.; Martinez-Roman, J.; Rubén Puche-Panadero; Riera-Guasp, M. (2016). Internal inductance of a conductor of rectangular cross-section using the proper generalized decomposition. COMPEL The International Journal for Computation and Mathematics in Electrical and Electronic Engineering. 35(6):2007-2021. doi:10.1108/COMPEL-03-2016-0124

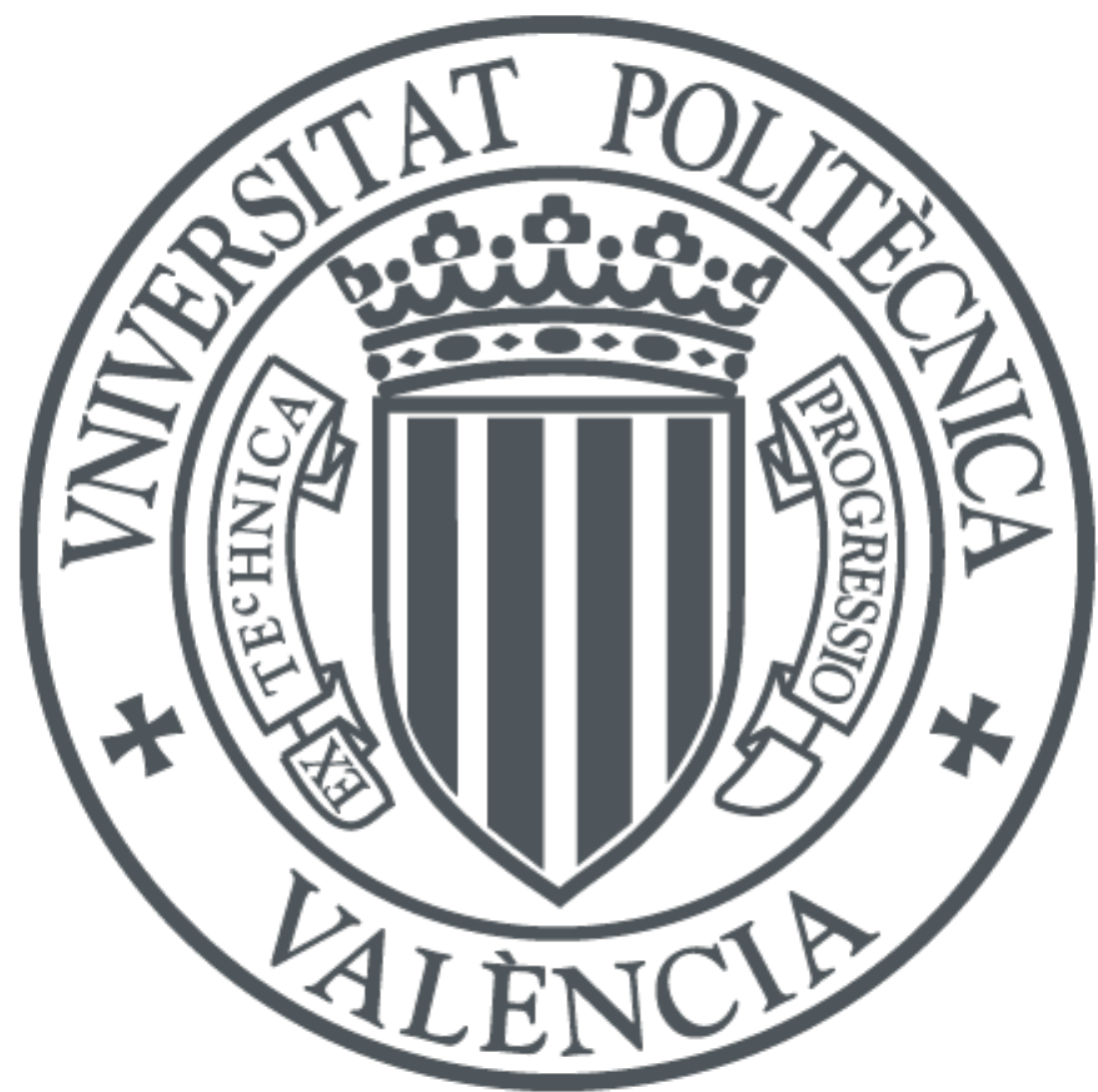

The final publication is available at

https://doi.org/10.1108/COMPEL-03-2016-0124

Copyright Emerald

Additional Information 


\title{
Internal inductance of a conductor of rectangular cross section using the Proper Generalized Decomposition
}

\begin{abstract}
$\underline{\text { Abstract }}$
Purpose

-Rectangular conductors play an important role in planar transmission line structures, multiconductor transmission lines, in power transmission and distribution systems, LCL filters, transformers, industrial busbars, MEMs devices, among many others. The precise determination of the inductance of such conductors is necessary for its design and optimization, but no explicit solution for the ac resistance and internal inductances per-unit length of a linear conductor with a rectangular cross section has been found, so numerical methods must be used. This paper introduces the use of a novel numerical technique, the Proper Generalized Decomposition (PGD), for the calculation of dc and ac internal inductances of rectangular conductors.
\end{abstract}

\section{Design/methodology/approach}

- The PGD approach is used to obtain numerically the internal inductance of a conductor with circular cross section and with rectangular cross section, both under dc and ac conditions, using a separated representation of the magnetic vector potential in a 2D domain. The results are compared with the analytical and approximate expressions available in the technical literature, with an excellent concordance.

\begin{abstract}
Findings
- The PGD uses simple one-dimensional meshes, one per dimension, so the use of computational resources is very low, and the simulation speed is very high. Besides, the application of the PGD to conductors with rectangular cross section is particularly advantageous, because rectangular shapes can be represented with a very few number of independent terms, which makes the code very simple and compact. Finally, a key advantage of the PGD is that some parameters of the numerical model can be considered as additional dimensions. In this paper, the frequency has been considered as an additional dimension, and the internal inductance of a rectangular conductor has been computed for the whole range of frequencies desired using a single numerical simulation.
\end{abstract}

\begin{abstract}
Originality/value
- The PGD is a promising new numerical procedure that has been applied successfully in different fields. In this paper, this novel technique is applied to find the dc and ac internal inductance of a conductor with rectangular cross section, using very dense and large one-dimensional meshes. The proposed method requires very limited memory resources, is very fast, can be programmed using a very simple code, and gives the value of the ac inductance for a complete range of frequencies in a single simulation. The proposed approach can be extended to arbitrary conductor shapes and complex multiconductor lines to further exploit the advantages of the PGD.
\end{abstract}

\section{Introduction}

Rectangular conductors play an important role in planar transmission line structures, such as microstrip lines, coplanar strips, signal traces of printed circuit board (Holloway and Kuester, 2009), (Matsuki and Matsushima, 2012), in multiconductor transmission lines (Antonini et al., 1999), in power transmission and distribution systems (Morgan, 2013), (Brito et al., 2016), LCL filters used in high power variable speed drives (VSDs) and grid interconnections (Gohil et al., 2016), in transformers (Moghaddami et al., 2016), in industrial busbars used in switchboards, distribution boards, or substation installations (Faiz et al., 2016), in electromagnetic devices equipped with flat coils (Rainey et al., 2007), in MEMs devices (Peters and Manoli, 2008), among many others. The precise determination of the inductance of such conductors is necessary for its design and optimization (Martinez et al., 2014).

Analytical solutions of the magnetic field and inductance of conductors are only available for the simplest shapes of cross sections, circular conductors (Morgan, 2013; Smith, 2014) and infinitely wide flat sheets (Berleze and Robert, 2003). For other shapes, such as the rectangular, triangular, or elliptical, only approximate expressions are available (Antonini et al., 1999; De Smedt, 2014; Freitas 
et al., 2015; Holloway and Kuester, 2009; Holloway et al., 2013; Matsuki and Matsushima, 2012; Morgan, 2013; Tsiboukis and Kriezis, 1983). In this case, different techniques have been proposed in the technical literature for computing the inductance of the conductor, such as direct methods of the functional analysis in (Tsiboukis and Kriezis, 1983), analytic solutions (Martinez et al., 2014), (Högas et al., 2015), (Brandao Faria and Raven, 2013), or semi analytical approximations (De Smedt, 2014), (Chassart et al., 2013). Nevertheless, one of the most used approaches has been the application of numerical techniques based in computational electromagnetics, which can be applied to conductors or arbitrary shapes. Integral equation methods have been proposed, for example in (Matsuki and Matsushima, 2012), and FEM models have been used in (Brito et al., 2016; Freitas et al., 2015; Faiz et al., 2016; Moghaddami et al., 2016). Nevertheless, the implementation of numerical methods has several drawbacks. Dirichlet conditions are usually set at infinity outer boundaries. This is achieved in FEM methods using a circular air boundary far enough from the conductors (Moghaddami et al., 2016), (Riba, 2015), which results in large domains to be meshed, or using special semi-infinite elements (Cardenas and Ezekoye, 2015), which introduces an additional complexity in the FEM code. Another drawback in the case of complex shapes is that the mesh must be very dense around corners and other geometric shapes where a high gradient of the field is expected, which increases greatly the number of degrees of freedom of the model, and hence, the requirement of computational resources (storage and processing time).

To alleviate the aforementioned problems, a novel numerical technique, the proper generalized decomposition (PGD), has been proposed recently (Chinesta et al., 2013; Giner et al., 2013; PinedaSanchez et al., 2010), and is applied in this paper to the computation of the internal inductance of a conductor with a rectangular cross section. The PGD allows the simultaneous use of very large domains, to properly establish the Dirichlet conditions at the infinite outer boundaries, with very dense meshes, to reduce the numerical errors of the simulation, without the penalties associated to traditional FEM approaches, because the number of unknowns scales linearly with the number of dimensions, instead of exponentially. Instead of using a single, dense multidimensional mesh for representing the geometrical domain, the PGD uses simple one-dimensional meshes, one per dimension, so that the use of computational resources is very low, and the simulation speed is very high. Besides, the application of the PGD to conductors with rectangular cross section is particularly advantageous, because rectangular shapes can be represented with a very few number of terms, which makes the code very simple and compact. Finally, a key advantage of the PGD is that some parameters of the numerical model can be considered as additional dimensions (Ammar et al., 2013), together with the geometrical ones. In the case of the computation of the inductance of a conductor at different frequencies, a traditional FEM approach would imply to solve the same geometrical model for each desired frequency, as in (Chiesa and Gustavsen, 2014). Instead, in this paper, the frequency has been considered as an additional dimension, and the internal inductance of a rectangular conductor has been computed for the whole range of frequencies desired using a single numerical simulation.

The structure of this paper is as follows. In Section 2, the dc inductance of a conductor is defined, and the approximate expressions that have been proposed in the technical literature for the case of a rectangular conductor are presented. In Section 3, the PGD is applied to obtain the internal inductance of the conductor. First, the PGD is applied to a circular conductor, for which an analytical closed expression exits, and second, it is applied to a rectangular conductor. In both cases the concordance of the PGD results and the existing ones is excellent. In Section 4, the use of the PGD is extended to compute the internal inductance and resistance under ac conditions. In this section, the frequency is treated as an additional dimension, so that a single simulation gives the ac resistance and internal inductance of the conductor for a whole frequency range. The PGD results are again validated using a circular conductor, for which a closed solution exits. Finally, Section 5 presents the conclusion of the paper. 


\section{DC internal inductance of a conductor}

The internal inductance $L_{i}$ per unit length of an infinitely long conductor of arbitrary shape is given by (Tsiboukis and Kriezis, 1983)

$$
\frac{L_{i}}{l}=\frac{1}{I^{2}} \iint \vec{B} \cdot \vec{H} d S=\frac{1}{\mu_{0} I^{2}} \iint \vec{B} \cdot \vec{B} d S=\frac{1}{\mu_{0} I^{2}} \int_{S}\left(\mathrm{~B}_{\mathrm{x}}{ }^{2}+\mathrm{B}_{\mathrm{y}}{ }^{2}\right) d S
$$

where $I$ is the current, the integral is carried out over the cross section of the conductor, and it has been assumed that the permeability $\mu_{0}$ is constant throughout the conductor. Using a 2D model, and making use of the magnetic vector potential (MVP), which under this conditions has only one component, $A_{\mathrm{z}}$, directed along the direction of the current, the components of the magnetic induction $\bar{B}$ in (1) can be obtained as

$$
\vec{B}=\nabla \mathrm{x} \overrightarrow{\mathrm{A}} \quad \Rightarrow \quad B_{x}=\frac{\partial}{\partial y} A_{z} \quad \text { and } \quad B_{y}=-\frac{\partial}{\partial x} A_{z}
$$

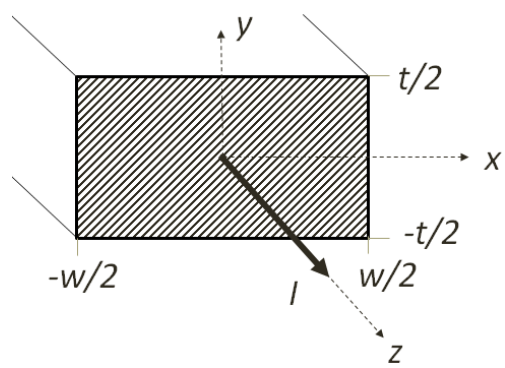

Fig. 1. Conductor with rectangular cross section parallel to the $z$-axis

In the case of an infinitely long conductor with a rectangular cross section, fed with a constant, dc current $I$, as represented in Fig. 1, the MVP can be obtained by superposition of the MVP generated by the elementary, infinitesimally thin subconductors in which the rectangular conductor can be partitioned, all with the same current density, as in (Berleze and Robert, 2003). In this case, the following integral must be solved

$$
A_{z}=\frac{\mu_{0} \cdot I}{2 \cdot \pi \cdot w \cdot t} \int_{-t / 2}^{t / 2} \int_{-\frac{w}{2}}^{\frac{w}{2}} \ln \left[\left(x-x^{\prime}\right)^{2}+\left(y-y^{\prime}\right)^{2}\right] d x^{\prime} d y^{\prime}+C
$$

Performing the derivatives of (2) directly inside the integral in (3), the components of the magnetic induction $\bar{B}$ are given by

$$
\begin{gathered}
B_{x}=\frac{\mu_{0} I}{2 \pi w t} \int_{-\frac{t}{2}}^{\frac{t}{2}} \int_{-\frac{w}{2}}^{\frac{w}{2}} \frac{\left(y-y^{\prime}\right)}{\left(x-x^{\prime}\right)^{2}+\left(y-y^{\prime}\right)^{2}} d x^{\prime} d y^{\prime} \\
B_{y}=-\frac{\mu_{0} I}{2 \pi w t} \int_{-t / 2}^{t / 2} \int_{-\frac{w}{2}}^{\frac{w}{2}} \frac{\left(x-x^{\prime}\right)}{\left(x-x^{\prime}\right)^{2}+\left(y-y^{\prime}\right)^{2}} d x^{\prime} d y^{\prime}
\end{gathered}
$$

and, from (1) and (4), the final expression of (1) is given by (Holloway et al., 2009)

$$
\frac{L_{i d c}}{l}=\frac{\mu}{(2 \pi w t)^{2}} \int_{-t / 2}^{t / 2} \int_{-w / 2}^{w / 2}\left[W_{1}^{2}+W_{2}^{2}\right] d x d y, \quad \text { with }
$$




$$
\begin{aligned}
& W_{1}=\left(\frac{w+2 x}{4}\right) \ln \left[\frac{\left(\frac{w}{2}+x\right)^{2}+\left(\frac{t}{2}-y\right)^{2}}{\left(\frac{w}{2}+x\right)^{2}+\left(\frac{t}{2}+y\right)^{2}}\right]+\left(\frac{w-2 x}{4}\right) \ln \left[\frac{\left(\frac{w}{2}-x\right)^{2}+\left(\frac{t}{2}-y\right)^{2}}{\left(\frac{w}{2}-x\right)^{2}+\left(\frac{t}{2}+y\right)^{2}}\right]+ \\
& +\left(\frac{t}{2}-y\right)\left[\tan ^{-1}\left(\frac{w-2 x}{t-2 y}\right)+\tan ^{-1}\left(\frac{w+2 x}{t-2 y}\right)\right]-\left(\frac{t}{2}+y\right)\left[\tan ^{-1}\left(\frac{w-2 x}{t+2 y}\right)+\tan ^{-1}\left(\frac{w+2 x}{t+2 y}\right)\right] \\
& W_{2}=\left(\frac{t+2 y}{4}\right) \ln \left[\frac{\left(\frac{w}{2}-x\right)^{2}+\left(\frac{t}{2}+y\right)^{2}}{\left(\frac{w}{2}+x\right)^{2}+\left(\frac{t}{2}+y\right)^{2}}\right]+\left(\frac{t-2 y}{4}\right) \ln \left[\frac{\left(\frac{w}{2}-x\right)^{2}+\left(\frac{t}{2}-y\right)^{2}}{\left(\frac{w}{2}+x\right)^{2}+\left(\frac{t}{2}-y\right)^{2}}\right]+ \\
& +\left(\frac{w}{2}-x\right)\left[\tan ^{-1}\left(\frac{t-2 y}{w-2 x}\right)+\tan ^{-1}\left(\frac{t+2 y}{w-2 x}\right)\right]-\left(\frac{w}{2}+x\right)\left[\tan ^{-1}\left(\frac{t-2 y}{w+2 x}\right)+\tan ^{-1}\left(\frac{t+2 y}{w+2 x}\right)\right]
\end{aligned}
$$

As (Holloway et al., 2009) and (Morgan, 2013) point out, the integral (5) cannot be evaluated in closed form, because it results in polylogarithms, which cannot be expressed in terms of elementary functions. So, numerical integration of (5) is needed to compute the dc inductance of the rectangular conductor. To avoid this drawback, approximate formula have been proposed in (Holloway et al., 2009) for the case of a small $t / w$ ratio, and similar formulae have been derived in (Morgan, 2013), among many others. One additional problem is that the integral that give the internal inductance of a conductor must be recomputed for each new shape of the conductor, as done in (Morgan, 2013) for elliptical and triangular cross sections.

\section{Proposed approach to obtain the de internal inductance of a conductor using the PGD}

In this paper, the proposed method for obtaining the dc inductance of a rectangular conductor is based on solving the diffusion equation of the MVP of the conductor, fed with a constant dc current,

$$
\frac{\partial^{2} A_{z}(x, y)}{\partial x^{2}}+\frac{\partial^{2} A_{z}(x, y)}{\partial y^{2}}=-\mu_{0} \cdot J_{0}(x, y) \quad \text { with }\left.A_{z}\right|_{x=\infty}=\left.A_{z}\right|_{y=\infty}=0
$$

where $J_{0}$ is the density current through the conductor cross section. Using the PGD approach (Chinesta et al., 2013, 2011), the 2D MVP $A_{z}(x, y)$ is expressed as a sum of products of elementary one dimensional functions:

$$
A_{z}(x, y)=\sum_{i=1}^{n} X_{i}(x) \cdot Y_{i}(y)
$$

where $n$ is the number of products, or modes, that approximate $A_{z}(x, y)$ with the desired precision. These modes are computed by the PGD algorithm using an iterative procedure which requires, at each step, the solution of simple 1D partial differential equations (PDEs), which makes the algorithm very fast. A brief explanation of the PGD applied to the solution of (6) is given in this section, although a detailed explanation of the PGD algorithm can be found in (Chinesta et al., 2013, 2011).

The basis of the PGD approach is to work with separated representations of the MVP (7), unknown, and also of the imposed current density $J_{0}(x, y)$, whose shape is the same as the conductor shape, 


$$
J_{0}(x, y)=\sum_{j=1}^{m} J x_{j}(x) \cdot J y_{j}(y)
$$

To be able to solve (6) numerically, the boundary condition at infinity in (6) is replaced by a boundary condition on a finite domain, $\Omega=\Omega_{\mathrm{x}} \times \Omega_{\mathrm{y}}=(-L, L) \times(-L, L)$, so that $L$ is much greater than the dimensions of the conductor (in this work $L$ is 100 times the width of the conductor). Using (7) and (8), (6) can be expressed as

$$
\sum_{i=1}^{n} \frac{\partial^{2} X_{i}(x)}{\partial x^{2}} Y_{i}(y)+\sum_{i=1}^{n} X_{i}(x) \frac{\partial^{2} Y_{i}(y)}{\partial y^{2}}=-\mu_{0} \sum_{j=1}^{m} J x_{j}(x) \cdot J y_{j}(y) \text { with }\left.A_{z}\right|_{x= \pm L}=\left.A_{z}\right|_{y= \pm L}=0
$$

The independent variables are no longer shown for simplicity. The functions $X_{i}$ and $Y_{i}$ can be computed numerically, using an iterative non-linear procedure. Supposing that the first $(n-1)$ modes have been computed, the $n$th mode, unknown, is obtained via a Galerkin procedure by replacing (7) in (6)

$$
\begin{aligned}
& A_{Z}=\sum_{i=1}^{n} X_{i} \cdot Y_{i}=\sum_{i=1}^{n-1} X_{i} \cdot Y_{i}+X_{n} \cdot Y_{n} \Rightarrow A_{Z}^{*}=X_{n}^{*} \cdot Y_{n}+X_{n} \cdot Y_{n}^{*} \\
& \int_{x=-L}^{x=L} \int_{y=-L}^{y=L} A_{Z}^{*}\left[\sum_{i=1}^{n}\left(\frac{\partial^{2} X_{i}}{\partial x^{2}} Y_{i}+X_{i} \frac{\partial^{2} Y_{i}}{\partial y^{2}}\right)+\mu_{o} \sum_{j=1}^{m} J x_{j} \cdot J y_{j}\right] d x d y=0
\end{aligned}
$$

To find the mode $n$ an iterative procedure is followed. Suppose that the function $Y_{n}$ is known at a given iteration, that is, $A_{z}^{*}=X_{n}^{*} \cdot Y_{n}$. The substitution of (10) in (11) gives

$$
\int_{y=-L}^{y=L} X_{n}^{*}(\sum_{i=1}^{n} \underbrace{\left(\int_{y=-L}^{y=L} Y_{n} \frac{\partial^{2} Y_{i}}{\partial y^{2}} d y\right)}_{\alpha_{i}} X_{i}+\sum_{i=1}^{n}(\underbrace{\left(\int_{y=-L}^{y=L} Y_{n} Y_{i} d y\right)}_{\beta_{i}} \frac{\partial^{2} X_{i}}{\partial x^{2}}+\sum_{j=1}^{m} \underbrace{\left(\int_{y=-L}^{y=L} Y_{n} J y_{j} d y\right)}_{\gamma_{j}} J x_{j}) d x=0
$$

This corresponds to the PDE

$$
\sum_{i=1}^{n} \alpha_{i} \cdot X_{i}+\sum_{i=1}^{n} \beta_{i} \cdot \frac{\partial^{2} X_{i}}{\partial x^{2}}+\sum_{j=1}^{m} \gamma_{j} \cdot J x_{j}=0
$$

And, passing all the known terms to the right hand side (RHS), gives

$$
\alpha_{n} \cdot X_{n}+\beta_{n} \cdot \frac{\partial^{2} X_{n}}{\partial x^{2}}=-\sum_{i=1}^{n-1}\left(\alpha_{i} \cdot X_{i}+\beta_{i} \cdot \frac{\partial^{2} X_{i}}{\partial x^{2}}\right)-\sum_{j=1}^{m}\left(\gamma_{j} \cdot J x_{j}\right)
$$

This equation is solved in the $x$ domain with a simple 1D finite differences or a 1D FE method, giving the value of $X_{n}$ in the present iteration. With this value, the computation of a new value of $Y_{n}$ is performed as 
$\int_{y=-L}^{y=L} Y_{n}^{*}(\sum_{i=1}^{n} \underbrace{\left.\int_{x=-L}^{x=L} X_{n} \frac{\partial^{2} X_{i}}{\partial x^{2}} d x\right)}_{\alpha_{i}^{\prime}} Y_{i}+\sum_{i=1}^{n} \underbrace{\left(\int_{x=-L}^{x=L} X_{n} X_{i} d x\right)}_{\beta_{i}^{\prime}} \frac{\partial^{2} Y_{i}}{\partial y^{2}}+\sum_{j=1}^{m} \underbrace{\left(\int_{x=-L}^{x=L} X_{n} J x_{j} d x\right)}_{\gamma_{j}^{\prime}} J y_{j}) d y=0$

This corresponds to the PDE

$$
\sum_{i=1}^{n} \alpha_{i}^{\prime} \cdot Y_{i}+\sum_{i=1}^{n} \beta_{i}^{\prime} \cdot \frac{\partial^{2} Y_{i}}{\partial y^{2}}+\sum_{j=1}^{m} \gamma_{j}^{\prime} \cdot J y_{j}=0
$$

and, passing all the known terms to the RHS, gives

$$
\alpha_{n}^{\prime} \cdot Y_{n}+\beta_{n}^{\prime} \cdot \frac{\partial^{2} Y_{n}}{\partial y^{2}}=-\sum_{i=1}^{n-1}\left(\alpha_{i}^{\prime} \cdot Y_{i}+\beta_{i}^{\prime} \cdot \frac{\partial^{2} Y_{i}}{\partial y^{2}}\right)-\sum_{j=1}^{m}\left(\gamma_{j}^{\prime} \cdot J y_{j}\right)
$$

This equation is solved in the $y$ domain with a simple 1D finite differences or a 1D FE method, giving the value of $Y_{n}$, finishing the present iteration. At every iteration the new values of $X_{n}$ and $Y_{n}$ are compared with the previous ones, and if the absolute value of their difference falls below a predefined threshold, the iterations are finished, and the solution is updated with the new values, corresponding to the $n$ mode. This iterative process begins again for computing the next $n+1$ mode. When the absolute value of the new mode falls below a predefined threshold, the solution is considered valid and the process finishes. The whole process starts assuming that no mode is known, that is, $n=0$.

Among the advantages of using the PGD for obtaining the internal inductance of the conductor with rectangular cross section, the following ones can be highlighted:

1. The 1D meshes used to obtain the 1D elementary functions $X_{i}(x)$ and $Y_{i}(y)$ in the PGD algortihm can be very large and dense. If a mesh of $N_{x}$ nodes is used for the $x$ variable, and $N_{y}$ nodes for the $y$ variable, the number of nodes of the mesh is not $N_{x} \cdot N_{y}$, as expected when solving the 2D problem (6), because only 1D PDEs must be solved using (14) and (17). This feature allows for the use of uniform meshes that are, at the same time, very large, to properly establish the boundary conditions $\left.A_{z}\right|_{x=\infty}=\left.A_{z}\right|_{y=\infty}=0$, and very dense, to reach a high precission in the computation of the internal inductance. The use of uniform meshes simplifies the solution of the problem, and in this paper the 1D PDEs have been solved using a simple finite difference method.

2. In (9) not only the MVP $A_{z}(x, y)$ is expressed as a separated representation, but also the imposed density current $J_{o}(x, y)=\sum_{j=1}^{m} J x_{j}(x) \cdot J y_{j}(y)$ must be expressed as a sum of modes. Arbitrary cross sections shapes can be represented in separated form using the most significant components obtained with the singular value decomposition (SVD). This is a great strength of the proposed method, because, alongside with the use of uniform meshes indicated in the previous point, it implies that a unique implementation of PGD algorithm can deal with any shape of conductors, just changing the separated representation of the imposed current density, generated by the SVD of the conductor shape. The case of the rectangular shape is specially well suited to be expressed as a separated representation, because it can be represented using just one mode, and only a few modes are needed for complex shapes composed of rectangular shapes, as shown in Fig. 2. In fact, any arbitrary shape can be represented as a combination of rectangular strips.

3. To obtain the components of the magnetic induction (2) from the solution of the MVP in (6) it is not necessary to compute the full 2D representation of (7). Instead, the derivatives in (2) can be made directly using the separated 1D representation of (7), which allows to perform the integral (1) using simple 1D domains. 


$$
B_{x}=\frac{\partial A_{z}(x, y)}{\partial y}=\sum_{i=1}^{n} X_{i}(x) \cdot \frac{\partial Y_{i}(y)}{\partial y} \quad \text { and } B_{y}(x, y)=-\frac{\partial A_{z}(x, y)}{\partial x}=-\sum_{i=1}^{n} \frac{\partial X_{i}(x)}{\partial x} \cdot Y_{i}(y)
$$
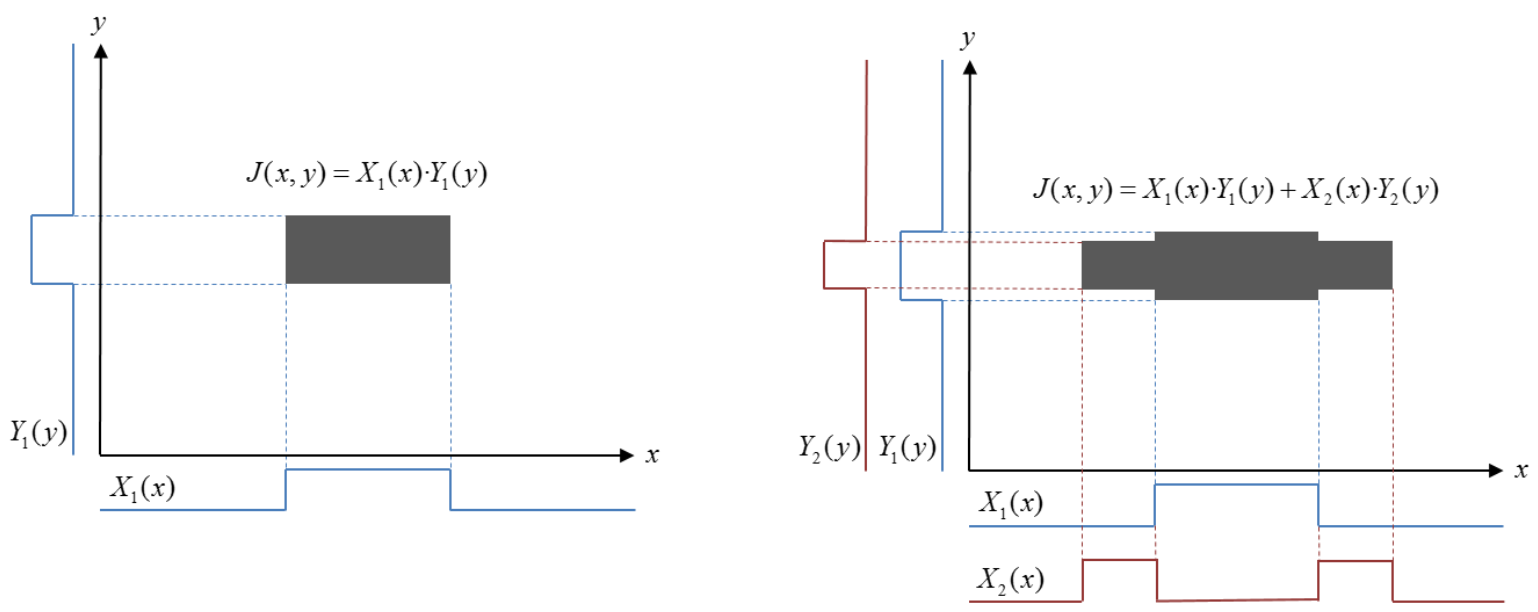

Figure 2. Separated representation of a conductor with rectangular cross section (one mode, left), and with a more complex shape made of rectangular components (two modes, right).

The proposed approach has been applied first to a circular conductor for which there exists an analytical solution, and after to a rectangular conductor with variable $t / w$ ratio.

\section{$\underline{\text { 3.1. DC inductance of a conductor with circular cross section }}$}

In the case of a copper wire with circular cross section, the internal inductance is equal to $L_{i d c} / l=$ $\mu_{o} / 8 \pi=50 \mathrm{nH} / \mathrm{m}$. The conductor simulated has a diameter of $20 \mathrm{~mm}$, and is placed at the centre of a $2 \mathrm{~m} \times 2 \mathrm{~m}$ domain. The domain height and width have been chosen to be 100 times the diameter of the conductor, to establish the boundary conditions at infinity. A uniform mesh of 100000 nodes has been used for discretizing each spatial dimension. 118 modes have been used to obtain the circular cross section in separated form. Fig. 3 shows the separated representation of the conductor and the MVP generated when it is fed with a constant dc current of $1000 \mathrm{~A} / \mathrm{m}^{2}$. The inductance of this conductor obtained with the proposed method is $49.999 \mathrm{nH}$.
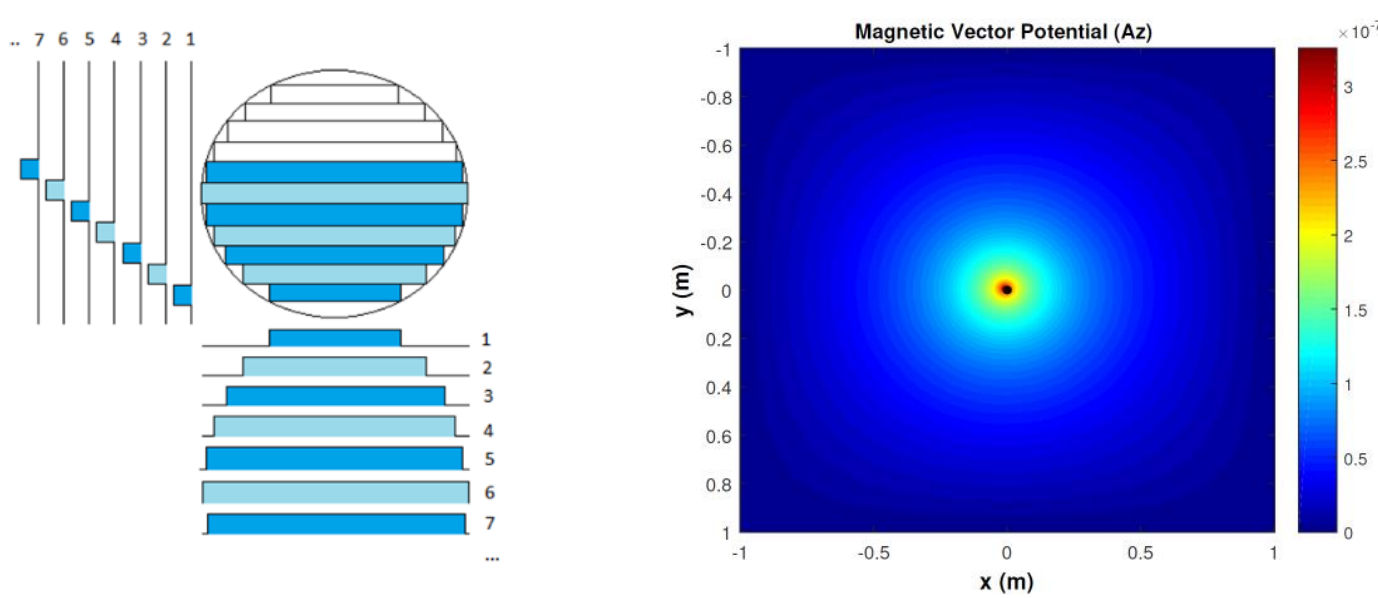

Figure 3. Left: separated representation of the circular cross section shape, with a diameter of $20 \mathrm{~mm}$, using rectangular strips. Right: MVP generated by the conductor, fed with a constant current $\left(1000 \mathrm{~A} / \mathrm{m}^{2}\right)$, obtained using the PGD with a rectangular domain $(2 \mathrm{~m} \times 2 \mathrm{~m})$, which has been discretized using a uniform mesh for each spatial dimension $\left(10^{5}\right.$ nodes each mesh). 
Fig. 4 shows a zoomed view of the MVP generated in the vicinity of the conductor, and also the modulus of the magnetic induction in the same area. The magnetic induction shown in Fig. 4, left, has been obtained using (18).
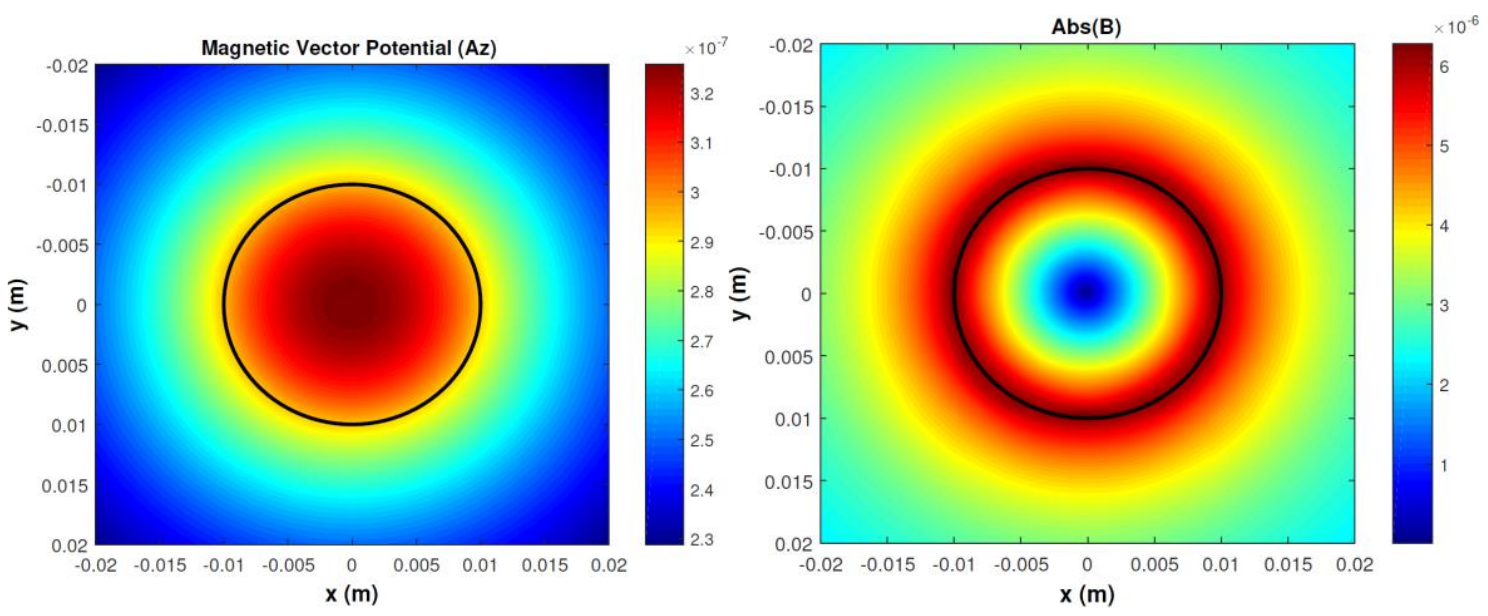

Figure 4. Left: zoomed view of the MVP displayed in Fig. 3 in the vicinity of conductor. Right: modulus of the magnetic induction, obtained from (18). The limits or the conductor are displayed using a thick, black line.

The MVP generated by the PGD algorithm as the solution to (6) is obtained in separate representation form, as given by (7). Fig. 5 shows the $X_{i}(x)$ functions used to build up the modes of the MVP in (7). Due to the symmetry of the model, the $Y_{i}(y)$ functions are equal to the $X_{i}(x)$ functions, so they are not shown in Fig. 5. Each one of these functions has $10^{5}$ values, so the total solution presented in Fig. 3, right, has been generated using a total of $2 \cdot 10 \cdot 10^{5}$ values. An equivalent solution using a full 2D mesh of the domain, with a mesh for each axis equal to the ones used in this work, requires a total number of values of to $10^{10}$, instead of the $2 \cdot 10^{6}$ values used by the PGD. Nevertheless, it is also important to remark that, separately, none of the functions $X_{i}(x)$ and $Y_{i}(y)$ that form the modes (7) have any physical meaning. Only their added products, (7), has a physical meaning, the MVP which is generated by the conductor.
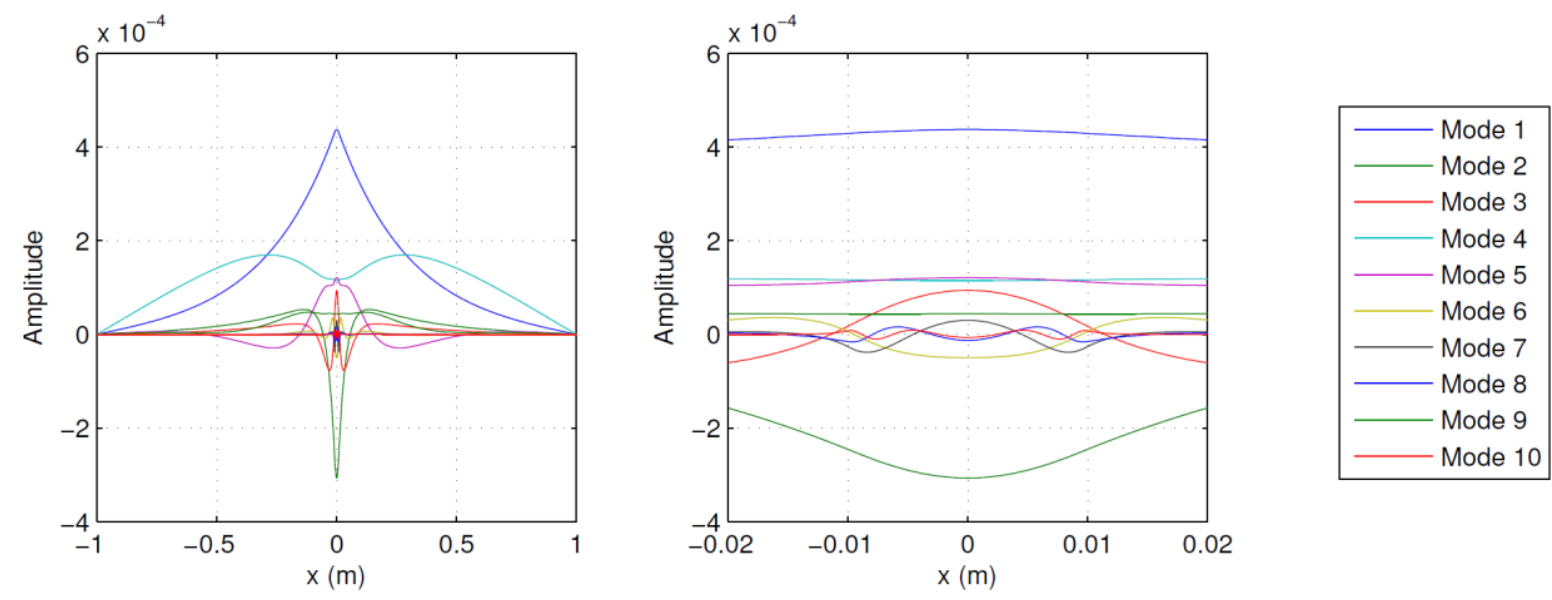

Figure 5. Modes of the MVP (7) generated by the conductor of circular cross section obtained using the PGD. Left: $X_{i}(x)$ functions for the whole domain. Right: $X_{i}(x)$ functions in the vicinity of the conductor. Due to the symmetry of the conductor, the $Y_{i}(y)$ functions have the same shape as the $X_{i}(x)$ functions, so they are not represented in the figure. 


\subsection{DC inductance of a conductor with rectangular cross section}

The inductance of a conductor with a rectangular cross section has been obtained using the proposed method for different $t / w$ ratios, and the results have been compared with the theoretical values given by (5), which has been integrated numerically. Fig 6 shows the results obtained with the PGD after solving (6) for the rectangular conductor, the MVP generated by the conductor (Fig. 6, left) and the modulus of the magnetic induction (Fig. 6, right).
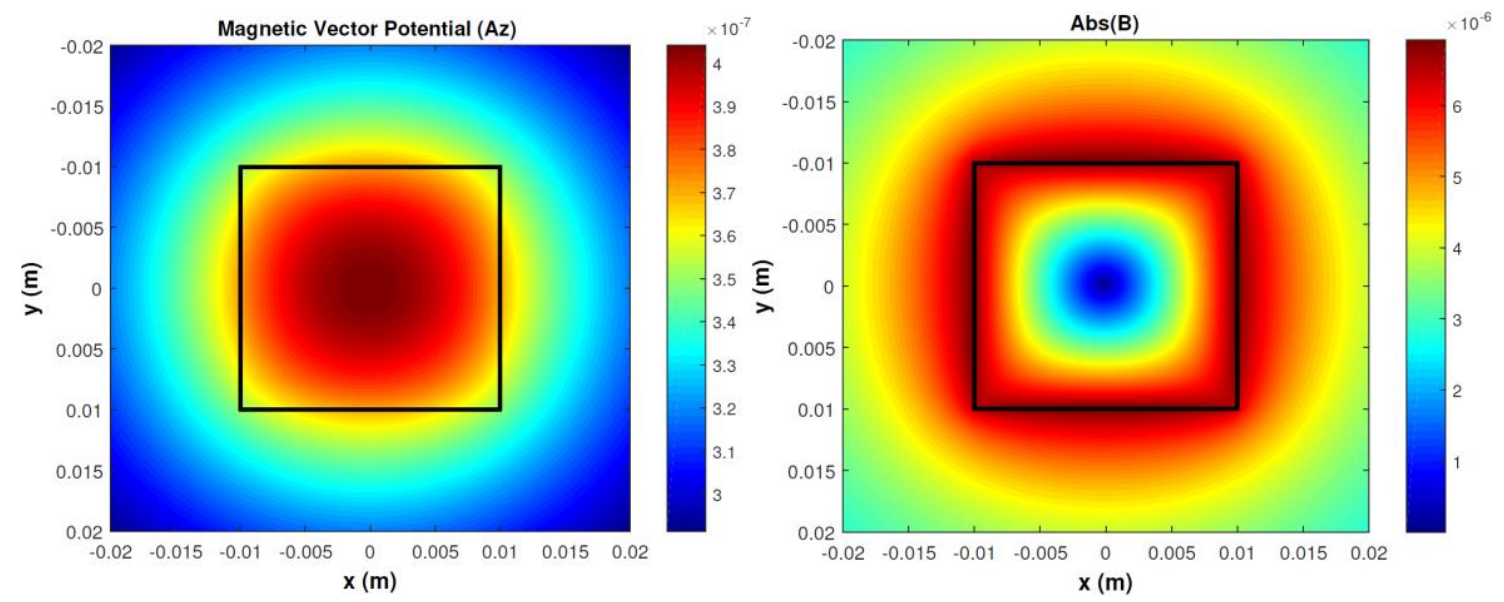

Figure 6. Left: MVP generated by a conductor with square cross section, (side length $0.02 \mathrm{~m}$ ), fed with a constant current $\left(1000 \mathrm{~A} / \mathrm{m}^{2}\right)$, obtained using the PGD with a rectangular domain $(2 \mathrm{~m} \mathrm{x} 2 \mathrm{~m})$, which has been discretized using a uniform mesh for each spatial dimension $\left(10^{5}\right.$ nodes each mesh). Right: modulus of the magnetic induction generated by the conductor, obtained from (18). The limits or the conductor are displayed using a thick, black line.

The internal dc inductance of the conductor with rectangular cross section has ben obtained for different $t / w$ ratios, and a plot of the results is presented in Fig. 7, along with the results obtained by numerical integration of (5).

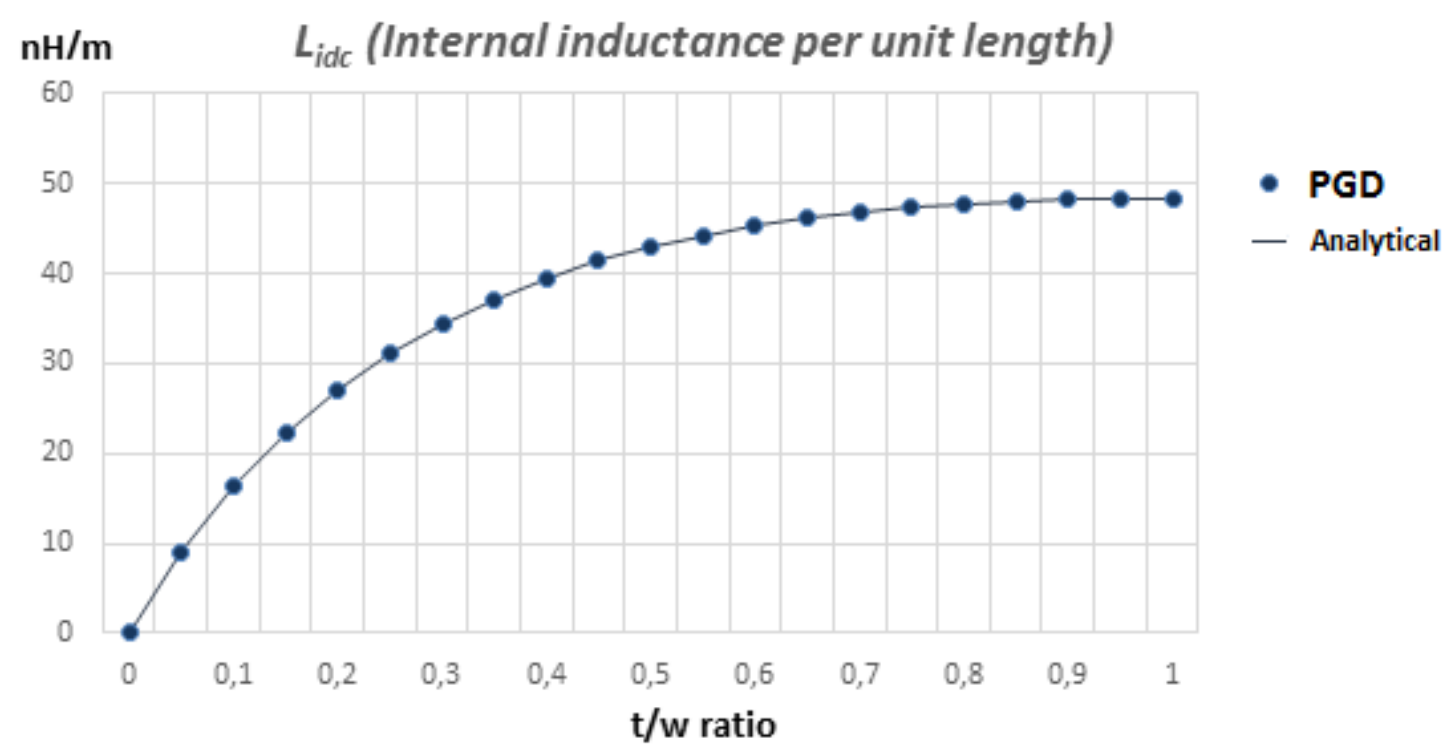

Figure 7. DC internal inductance $L_{i d c}$ of a conductor with a rectangular cross section, for different aspect ratio. ratio $t / w$, computed using the analytical expression (5), and with the proposed approach. 


\section{Proposed approach to obtain the ac internal inductance of a conductor using the PGD}

One of the advantages of the proposed method, besides the fact that it can be applied to complex shapes of conductors for whom no analytical formula such as (5) is available, is that it can be easily extended to obtain the internal inductance and resistance for any frequency, that is, taking into account the skin effect, which increases the resistance of the conductor and decreases its internal inductance (Chiesa and Gustavsen, 2014). As (Morgan, 2013) points out, no explicit solution for the ac resistance and internal inductances per-unit length of a linear conductor with a rectangular cross section has been found, so numerical methods must be used.

To take into account the skin effect, at any frequency $f$, (6) is replaced by

$$
\frac{\partial^{2} A_{z}(x, y, f)}{\partial x^{2}}+\frac{\partial^{2} A_{z}(x, y, f)}{\partial y^{2}}=-\mu_{0} \cdot J=-\mu_{0} \cdot\left(J_{0}(x, y)-j \cdot \omega \cdot \sigma(x, y) \cdot A(x, y, f)\right)
$$

where $\omega(=2 \cdot \pi \cdot f)$ is the frequency in radians per second, $J_{0}$ is the imposed current, and $J$ is the total current density. Besides, as an additional advantage of using the PGD to obtain the internal ac inductance, the problem domain has been extended to include the frequency as an additional dimension,

$$
A(x, y, f)=\sum_{i=1}^{n} X_{i}(x) \cdot Y_{i}(y) \cdot F_{i}(f)
$$

where $F_{i}(f)$ is a function of the frequency of the current source. In this way, instead of solving repeatedly the diffusion equation for each desired frequency, a single solution is found for (19), using a 3D MVP (20): two spatial dimensions and one frequency dimension. And adding the frequency as a parametric dimension can be done with a negligible computational cost (Chinesta et al., 2013). The proposed method has been applied to compute the resistance and the internal inductance of the circular and rectangular conductors of the previous section.

\subsection{AC inductance of a conductor with circular cross section}

The relation between the values of the dc resistance and dc internal inductance of a circular conductor, of radius $r_{s}$ and resistivity $\rho,\left(R_{d c}, L_{i d c}\right)$ and their ac values for a given frequency $f$, $\left(R_{i a c}, L_{i a c}\right)$ are available in closed form, as

$$
\begin{aligned}
\frac{L_{i a c}}{L_{i d c}} & =\frac{4}{m \cdot r_{s}}\left[\frac{\operatorname{ber}\left(m \cdot r_{s}\right) \cdot \operatorname{ber}^{\prime}\left(m \cdot r_{s}\right)-b e i\left(m \cdot r_{s}\right) \cdot b e i^{\prime}\left(m \cdot r_{s}\right)}{\left(b e r^{\prime}\left(m \cdot r_{s}\right)\right)^{2}+\left(b e i^{\prime}\left(m \cdot r_{s}\right)\right)^{2}}\right] \\
\frac{R_{a c}}{R_{d c}} & =\frac{m \cdot r_{s}}{2}\left[\frac{\operatorname{ber}\left(m \cdot r_{s}\right) \cdot b e i^{\prime}\left(m \cdot r_{s}\right)-\operatorname{ber}^{\prime}\left(m \cdot r_{s}\right) \cdot b e i\left(m \cdot r_{s}\right)}{\left(\operatorname{ber}^{\prime}\left(m \cdot r_{s}\right)\right)^{2}+\left(b e i^{\prime}\left(m \cdot r_{s}\right)\right)^{2}}\right]
\end{aligned}
$$

where the parameter $m=\sqrt{2 \pi f \mu_{o} / \rho}$ depends on the frequency, and $\operatorname{ber}(x), \operatorname{bei}(x), \operatorname{ber}^{\prime}(x)$ y $b e i^{\prime}(x)$ are the Kelvin function of zero order. The ac resistance of the conductor is computed as $R_{a c}=\int_{S}|J|^{2} d S / \sigma|I|^{2}$, where $\mathrm{S}$ is the conductor cross section surface, $J$ is the current density and $I$ is the total current. Fig. 8 shows the comparison between (21) and the results obtained with the proposed approach for a copper, circular conductor with a diameter equal to $20 \mathrm{~mm}$. 

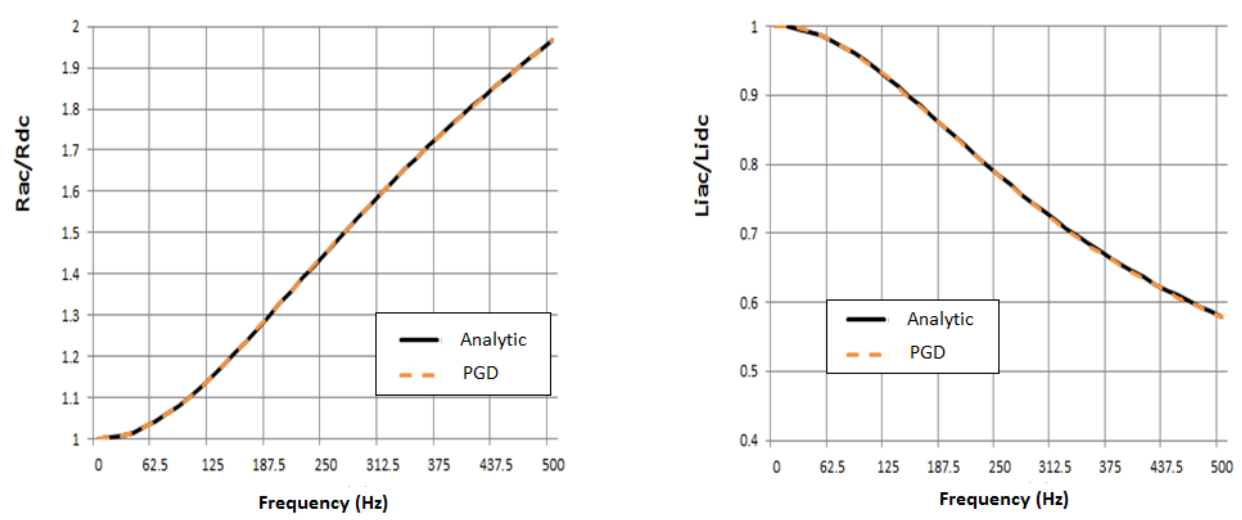

Figure 8. Left, ratio between the ac and the dc resistance $\left(R_{a c} / R_{d c}\right)$, and, right, ratio between the dc and the ac internal inductance $\left(L_{i a c} / L_{i d c}\right)$, for a circular conductor with a diameter of $20 \mathrm{~mm}$. Both the analytical values obtained from (21), solid line, and the values obtained using the proposed PGD approach, dotted line, have been represented.

\subsection{AC inductance of a conductor with rectangular cross section}

The proposed procedure has been applied to the computation of the ac resistance and the ac internal inductance of a conductor with a squared cross section of $1 \mathrm{~cm}^{2}$, and the results are shown in Fig. 9 .
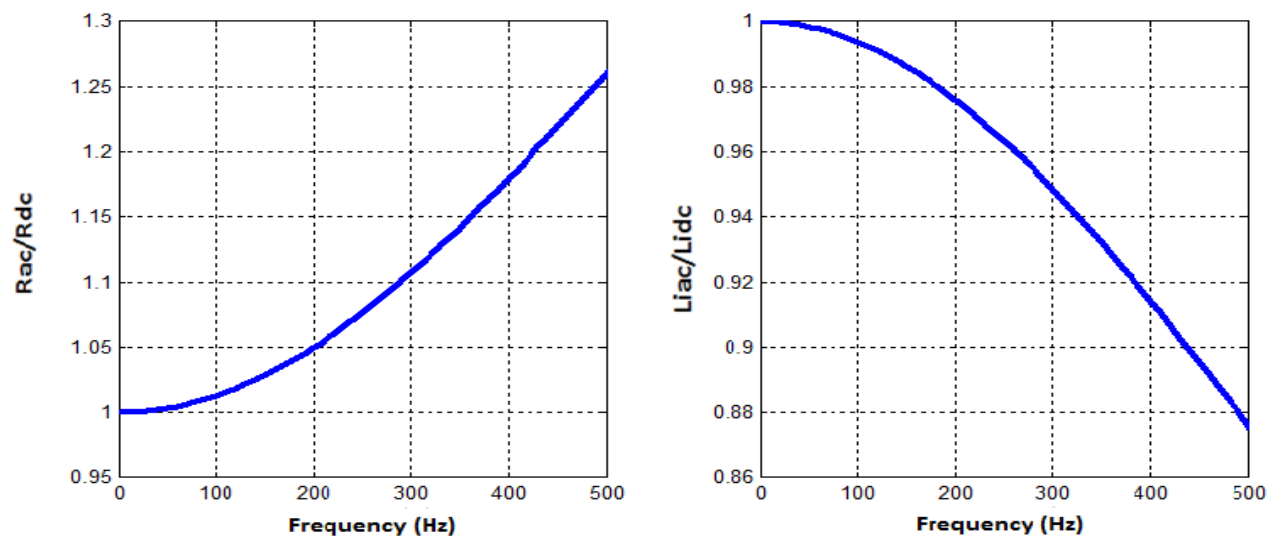

Figure 9. Left, ratio between the ac and the dc resistance $\left(R_{a c} / R_{d c}\right)$, and, right, ratio between the ac and the dc internal inductance $\left(L_{i a c} / L_{i d c}\right)$, for a square conductor with side length equal to $10 \mathrm{~mm}$, obtained using the proposed PGD approach.

\section{$\underline{\text { 5. Conclusions }}$}

In this paper, the PGD has been applied to compute the dc inductance of a conductor of rectangular cross section, as a function of the height/width ratio. The results have been found coincident with the numerical integration of the analytical formula available for this case, which validates the proposed approach. The dc inductance of a conductor with an arbitrary shape can be easily computed with the proposed approach, just introducing the separated representation of its shape in (9). And this representation can be obtained easily using the SVD. Besides, the proposed approach has been extended to obtain the ac values of the resistance and internal inductance of a circular and a square conductor, and the results obtained coincide with those available analytically. As an added benefit of the use of the PGD, the frequency has been introduced in the formulation as an additional dimension, 
so that the results obtained are available as a "virtual chart", which contains the solution for every frequency value within the range of frequencies selected. Following this approach, in a future work, the dimensions of the rectangular conductor will be introduced also as additional parametric dimensions, which will generate a single 3D solution (width, height and frequency) which contains the internal inductance and the resistance of a rectangular conductor for any frequency and for any dimensions of the conductor.

\section{References}

Ammar, A., Huerta, A., Chinesta, F., Cueto, E., Leygue, A., 2013. Parametric solutions involving geometry: A step towards efficient shape optimization. Comput. Methods Appl. Mech. Eng. 268, 178-193.

Antonini, G., Orlandi, A., Member, S., Paul, C.R., 1999. Internal Impedance of Conductors of Rectangular Cross Section. IEEE Trans. Microw. Theory Tech. 47, 979-985.

Berleze, S.L.M., Robert, R., 2003. Skin and proximity effects in nonmagnetic conductors. IEEE Trans. Educ. 46, 368-372.

Brandao Faria, J.A., Raven, M.S., 2013. On the success of electromagnetic analytical approaches to full timedomain formulation of skin effect phenomena. Prog. Electromagn. Res. M 31, $29-43$.

Brito, A.I., Machado, V.M., Almeida, M.E., Guerreiro Das Neves, M., 2016. Skin and proximity effects in the series-impedance of three-phase underground cables. Electr. Power Syst. Res. 130, 132-138.

Cardenas, D.E., Ezekoye, O.A., 2015. Thermal Characterization of Electrical Wires and Insulation Operated in Variable Frequency Mode. Fire Technol. 51, 1071-1092.

Chassart, C.D. de, Van Beneden, M., Kluyskens, V., Dehez, B., 2013. Semi-analytical determination of inductances in windings with axial and azimuthal wires. COMPEL Int. J. Comput. Math. Electr. Electron. Eng. 35, 2-15.

Chiesa, N., Gustavsen, B., 2014. Frequency-dependent modeling of transformer winding impedance from $\mathrm{R}(\omega) / \mathrm{L}$ measurements. IEEE Trans. Power Deliv. 29, 1511-1513.

Chinesta, F., Ladeveze, P., Cueto, E., 2011. A Short Review on Model Order Reduction Based on Proper Generalized Decomposition. Arch. Comput. Methods Eng. 18, 395-404.

Chinesta, F., Leygue, A., Bordeu, F., Aguado, J. V., Cueto, E., Gonzalez, D., Alfaro, I., Ammar, A., Huerta, A., 2013. PGD-Based Computational Vademecum for Efficient Design, Optimization and Control. Arch. Comput. Methods Eng. 20, 31-59.

De Smedt, R., 2014. Partial self inductance at DC of some common cross sections. 2014 18th IEEE Work. Signal Power Integrity, SPI 2014 - Proc. 2-5.

Freitas, D., Guerreiro Das Neves, M., Almeida, M.E., Malo Machado, V., 2015. Evaluation of the longitudinal parameters of an overhead transmission line with non-homogeneous cross section. Electr. Power Syst. Res. 119, 478-484.

Giner, E., Bognet, B., Ródenas, J.J., Leygue, A., Fuenmayor, F.J., Chinesta, F., 2013. The Proper Generalized Decomposition (PGD) as a numerical procedure to solve 3D cracked plates in linear elastic fracture mechanics. Int. J. Solids Struct. 50, 1710-1720.

Gohil, G., Bede, L., Teodorescu, R., Kerekes, T., Blaabjerg, F., 2016. An Integrated Inductor For Parallel Interleaved Three-Phase Voltage Source Converters. IEEE Trans. Power Electron. 31, 3400-3413.

Högas, M., Rydler, K.E., Stenarson, J., Yhland, K., 2015. Analytic Solution of the Magnetic Field and Inductance in a Coaxial Short Circuit. IEEE Trans. Instrum. Meas. 64, 1582-1587.

Holloway, C.L., Kuester, E.F., 2009. DC Internal Inductance for a Conductor of Rectangular Cross Section. Electromagn. Compat. IEEE Trans. 51, 338-344.

Holloway, C.L., Kuester, E.F., Ruehli, A.E., Fellow, L., Antonini, G., 2013. Partial and Internal Inductance: Two of Clayton R. Paul's Many Passions. IEEE Trans. Electromagn. Compat. 55, 600-613.

Holloway, C.L., Member, S., Kuester, E.F., 2009. DC Internal Inductance for a Conductor of Rectangular Cross Section. Electromagn. Compat. IEEE Trans. 51, 338-344.

J. Faiz , H. Ehya, A.M. Takbash, S. Shojaee, M. Hamidian, A.G., 2016. Recent progresses in bus-ducts design. COMPEL - Int. J. Comput. Math. Electr. Electron. Eng. 35, 117-136.

Martinez, J., Babic, S., Akyel, C., 2014. On evaluation of inductance, DC resistance, and capacitance of coaxial inductors at low frequencies. IEEE Trans. Magn. 50, 1-12.

Matsuki, M., Matsushima, A., 2012. Efficient impedance computation for multiconductor transmission lines of rectangular cross section. Prog. Electromagn. Res. B 43, 373-391.

Moghaddami, M., Moghadasi, A., Sarwat, A.I., 2016. An algorithm for fast calculation of short circuit forces in high current busbars of electric arc furnace transformers based on method of images. Electr. Power Syst. Res. 136, 173-180. 
Morgan, V.T., 2013. The current distribution, resistance and internal inductance of linear power system conductors-a review of explicit equations. IEEE Trans. Power Deliv. 28, 1252-1262.

Peters, C., Manoli, Y., 2008. Inductance calculation of planar multi-layer and multi-wire coils: An analytical approach. Sensors Actuators, A Phys. 145-146, 394-404.

Pineda-Sanchez, M., Chinesta, F., Roger-Folch, J., Riera-Guasp, M., Pérez-Cruz, J., Daïm, F., 2010. Simulation of skin effect via separated representations. COMPEL Int. J. Comput. Math. Electr. Electron. Eng. 29, 919-929.

Rainey, J.K., DeVries, J.S., Sykes, B.D., 2007. Estimation and measurement of flat or solenoidal coil inductance for radiofrequency NMR coil design. J. Magn. Reson. 187, $27-37$.

Riba, J.-R., 2015. Analysis of formulas to calculate the AC resistance of different conductors' configurations. Electr. Power Syst. Res. 127, 93-100.

Smith, G.S., 2014. A simple derivation for the skin effect in a round wire. Eur. J. Phys. 35, 25002-13.

Tsiboukis, T.D., Kriezis, E.E., 1983. Calculation of inductance of conductors with various shapes of cross section by direct methods of the functional analysis. Nuovo Cim. B Ser. 11 73, 177-188. 